\title{
Synergistic activity of doped zinc oxide nanoparticles with antibiotics: ciprofloxacin, ampicillin, fluconazole and amphotericin $B$ against pathogenic microorganisms
}

\author{
NEHA SHARMA ${ }^{1}$, SAVITA JANDAIK ${ }^{1}$ and SANJEEV KUMAR ${ }^{2}$ \\ ${ }^{1}$ Department of Biotechnology, Shoolini University, Bajhol, Post Office Sultanpur, Solan, HP-173212, India \\ ${ }^{2}$ Goswamy Ganesh Dutta Satnam Dharma (GGDSD) College, sector 32, Department of Physics, Chandigarh-160030, India
}

Manuscript received on September 24, 2015; accepted for publication on November 12, 2015

\begin{abstract}
Combination therapy of antibiotics and nanoparticles can be used against multi drug resistant microorganisms. Nanoparticles (NPs) have been reported to show antimicrobial activity. The antimicrobial activities of doped $\mathrm{ZnO}$ nanoparticles ( $\mathrm{ZnO} \mathrm{NPs}$ ) were studied against fungi, gram-positive and gramnegative bacteria using the standard microdilution method. The interaction between the nanoparticle and the antibiotic was estimated by calculating the fractional inhibitory concentration (FIC index) of the combination through checkerboard assay. Experimental results demonstrated that $10 \%$ doped zinc oxide nanoparticles ( $\mathrm{ZnO} \mathrm{NPs}$ ) exhibited the maximum antimicrobial effect in contrast with that of the $1 \%$ loading and pure $\mathrm{ZnO}$ nanoparticles. The enhancement in antimicrobial effect was seen when combined with antibiotic. Synergistic and additive effects were found. No antagonistic effect was found. More synergistic effect was observed when combined with ciprofloxacin than ampicillin. Fungus showed only additive effect. The results are quite in terms with MIC clearly depicting that high doping agent is most suitable for combined therapy. 100\% synergistic interaction was observed in higher doping with both ciprofloxacin and ampicillin. This study provides a preliminary report of the synergistic activity of nanoparticles with antibiotics against different pathogenic strains. This provides groundwork for further studies on the combination therapy of nanoparticles with antibiotics.
\end{abstract}

Key words: antimicrobial activity, MIC, ZnO nanoparticles, FIC, checkerboard assay.

\section{INTRODUCTION}

The increasing and indiscriminate usage of antibiotics and poor patient compliance has led to the development of bacterial immunity to antibiotics. Around the world, as much as $60 \%$ of hospital-acquired infections are caused by drugresistant microorganisms (Edmond et al. 1999). The organism has successfully developed numerous

Correspondence to: Savita Jandaik

E-mail: drsavitajandaik@gmail.com / neha1984micro@gmail.com strategies for resisting the action of practically all antibiotics (Kuroda et a1. 2001). Currently used antifungal agents also have toxic sideeffects, may interact with other drugs, and become ineffective as a consequence of the rapid growth of fungal resistance (Hammer et al. 1998, Shahi et al. 1999). Moreover, the therapeutic response may be slow, and thus inappropriate for treatment of patients with severe or rapidly progressive mycoses. This resistance to antimicrobial agents 
has resulted in morbidity and mortality from treatment failures, and increased health care costs. Although determining the precise public health risk and estimating the gain in prices is not a simple task, there is the slight question that the emerging antibiotic resistance is a serious worldwide problem. Likewise, the development of vaccines and new antimicrobial agents has not kept pace with resistance; therefore, the search for other methods of therapy, such as synergistic combinations, is necessary. Combination therapy is applied with the intention of expanding the antimicrobial spectrum, minimizing toxicity, preventing the emergence of resistant mutants during therapy and obtaining synergistic antimicrobial activity (Eliopoulos and Moellering Jr 1991). The increased clinical response to combination therapy is explained to be due to synergism between the antibiotics used. Synergism of a combination of antibiotics can be stated as fractional inhibitory concentration indices (FICi) derived from a checkerboard titration. Synergism has been defined as a phenomenon in which two different compounds are combined to enhance their individual activity. If the combination results in worsening effect, it is called antagonism. An effect which is less than synergistic but not antagonistic is termed as additive or indifference (Rani et al. 2009).

Nanotechnology represents a modern and innovative approach to develop new formulations based upon metallic nanoparticles with antimicrobial properties. A probe of the interactions of antibiotics with silver (Ag) nanoparticles is the most common among studies dedicated to the testing of combined action of nanoparticles with antibiotics. Few studies have found that the efficacy of antimicrobial agents can be improved by combining them with nanoparticles against different pathogens, including Staphylococcus aureus, Pseudomonas aeruginosa, Escherichia coli, etc. Recently, some metal nano particles have been valued for increasing the antibacterial actions of different antibiotics. Therapeutic roles for zinc in different diseases have been established in recent years. Zinc oxide has a very good potential to get into the clinic (Shopsin et al. 1999). Studies have revealed improved activity of nano $\mathrm{ZnO}$ when used in combination with cephalosporins, beta lactums and amino glycosides against different pathogenic microorganisms (Gaddad et al. 2010, Solomon et al. 2007).

The purposes of the present investigations were to evaluate the antimicrobial activity of the nanoparticles and compare them with the effect of antibiotics on the increasing resistance of different pathogenic microbes used; and to evaluate the interaction of the nanoparticles and antibiotics on these lines.

\section{MATERIALS AND METHODS}

Microrganisms: In the present study, six bacterial and two fungal isolates were procured from GianSagar Medical College, Rajpura, Punjab. The isolates were identified by conventional methods. Standard cultures (s) of bacteria and fungi were procured from Institute of Microbial Technology (IMTECH), Chandigarh. Following bacterial and fungal isolates were chosen:

Bacteria: Escherischia coli (MTCC 739), Klebsiella pneumoniae (MTCC 109), Pseudomonas aeruginosa (MTCC 741), Salmonella typhi (MTCC 98), Staphylococcus aureus (MTCC 737) and Bacillus subtilis (MTCC 736).

Fungi: Trichophyton mentagrophytes (MTCC 8476), Aspergillus fumigatus (MTCC 7136), Candida albicans (MTCC 227) and Cryptococcus neoformans (25:102(PGI) NCCPF).

Antimicrobial agents: Standard laboratory powders of Ciprofloxacin (Cip), ampicillin (Amp), flucanozole (Flu) and amphotericin B (Amp B) were used in the present studies. 
Nanoparticles: Pure and doped $\mathrm{ZnO}$ nanoparticles (10\% Fe, 10\% Mn, 10\% Cu, 10\% Co, $1 \%$ Fe, $1 \%$ $\mathrm{Mn}, 1 \% \mathrm{Cu}$ and $1 \% \mathrm{Co}$ ) with an average size of $20 \mathrm{~nm}$ were obtained from Department of Physics, Chitkara University, Chandigarh [characterized by Scanning Electron Microscopy (SEM), Transmission Electron Microscopy (TEM), X-Ray Diffraction (XRD) and Ultra Violet- Visible (UVVis) Spectroscopy].

Nanoparticles stock preparation: A stock suspension was prepared by suspending the nanoparticles in methanol to yield a final concentration of 100 $\mathrm{mg} / \mathrm{ml}$. This stock solution was then sonicated for 30 minutes of repeating the cycle after every 7 minutes. Every assay was done within 1-2 h of sonication. The suspension was kept at $4{ }^{\circ} \mathrm{C}$ and subjected to vigorous vortex mixing before assay.

Determination of minimum inhibitory concentration: Minimum inhibitory concentration (MIC) of the nanoparticles for bacteria was done by Broth micro dilution reference method (CLSI 2006, M7A7). The MIC of nanoparticles for filamentous fungi was done by Broth micro dilution reference method (CLSI 2008a, M38-A2) and for yeast by (CLSI 2008b, M27-A3). Resazurin was used as indicator. The lowest concentration that prevented color change was taken as a minimum inhibitory concentration.

Determination of interaction between nanoparticles and antibiotics: The combined effect between doped $\mathrm{ZnO}$ nanoparticle and standard antibiotics (Ampicillin, Ciprofloxacin, Amphotericin B and Fluconazole) was done by checkerboard assay in 96 well microtiter plate (Pillai et al. 2005). The concentration ranges for nanoparticles and antibiotics were $4 \mathrm{X} \mathrm{MIC-MIC/4}$. The inoculum contained $5 \times 10^{5} \mathrm{cfu} / \mathrm{ml}$. Antimicrobial solutions were prepared and freshly diluted on the day of the test. Each test was performed in triplicate. Fractional inhibitory concentrations (FICs) were calculated as: (MIC of drug A or B in combination) / (MIC of drug A or B alone), and the FIC index was obtained by adding the FIC values. FIC values were interpreted as synergistic if values were $\leq$ 0.5 , additive or indifferent to values $>0.5$ to 4.0 and antagonistic for values $>4.0$ (Braga et al. 2005, Odds 2003).

\section{RESULTS}

Minimum Inhibitory Concentration (MIC) (Table I):

Minimum Inhibitory Concentration range for undoped $\mathrm{ZnO}$ was $0.16-0.43 \mathrm{mg} / \mathrm{ml}$ in case of bacteria. The most effective value of MIC was obtained against $S$. typhi (s and c) and E. coli (s) at $0.16 \pm 0.01 \mathrm{mg} / \mathrm{ml}$ (s and c) and $0.16 \pm 0.02 \mathrm{mg} /$ $\mathrm{ml}$ concentrations respectively. MIC observed for T. mentegrophytes (s) and C. neoformens (c) was $0.33 \pm 0.14 \mathrm{mg} / \mathrm{ml}$ and $26.6 \pm 14.4 \mathrm{mg} / \mathrm{ml}$ respectively.

Excellent minimum inhibitory concentration of $0.09 \mathrm{mg} / \mathrm{ml}$ was obtained with $10 \%$ iron $(\mathrm{Fe})$ doping for B. subtilis (s) followed by $0.11 \mathrm{mg} / \mathrm{ml}$ for $E$. coli (s) and $0.12 \mathrm{mg} / \mathrm{ml}$ for $S$. aureus (c) at the same doping concentration. In case of $1 \%$ Fe doping, of all the pathogenic microbes used $S$. typhi (s and c) showed best results at $0.13 \mathrm{mg} / \mathrm{ml}$ minimum inhibitory concentration. MIC values for both the fungal strains, i.e. T. mentegrophytes (s) and $C$. neoformens (c) were $0.25 \pm 0.21 \mathrm{mg} / \mathrm{ml}$ and $11.5 \pm 11.8$ in case of $10 \% \mathrm{Fe}$ doping and $0.33 \pm 0.14$ $\mathrm{mg} / \mathrm{ml}$ and $13.5 \pm 10.9 \mathrm{mg} / \mathrm{ml}$ in case of $1 \% \mathrm{Fe}$ doping respectively.

For $10 \%$ doped manganese $(\mathrm{Mn})$, MIC range was $0.11-0.27 \mathrm{mg} / \mathrm{ml}$ and most operative MIC value was obtained at $0.11 \pm 0.02 \mathrm{mg} / \mathrm{ml}$ against S. typhi (s) whereas K. pneumonia (c) gave most effective MIC at a concentration of $0.13 \pm 0.01 \mathrm{mg} /$ $\mathrm{ml}$ with $1 \% \mathrm{Mn}$ doping. The highest values of MIC of $0.27 \pm 0.02$ and $0.28 \pm 0.23 \mathrm{mg} / \mathrm{ml}$ were obtained for P. aeruginosa (c) with both $10 \%$ and $1 \% \mathrm{Mn}$ 
TABLE I

Minimum inhibitory concentration $(\mathrm{mg} / \mathrm{ml})$ of pure $\mathrm{ZnO}$ and doped $\mathrm{ZnO}$ nanoparticle against various microorganisms. S: standard isolate, $\mathrm{C}$ : Clinical isolate.

\begin{tabular}{|c|c|c|c|c|c|c|}
\hline Microorganisms & Doping \% & $\mathrm{ZnO}$ & Fe doping & Mn doping & Co doping & Cu doping \\
\hline Bacteria & \multicolumn{6}{|c|}{ Concentration (mg/ml) } \\
\hline \multirow{2}{*}{ B. subtilis (S) } & 10 & \multirow{2}{*}{$0.21 \pm 0.06$} & $0.09 \pm 0.00$ & $0.19 \pm 0.00$ & $0.10 \pm 0.01$ & $0.004 \pm 0.00$ \\
\hline & 1 & & $0.26 \pm 0.09$ & $0.19 \pm 0.026$ & $0.33 \pm 0.033$ & $0.018 \pm 0.0$ \\
\hline \multirow{2}{*}{ B. subtilis $(\mathrm{C})$} & 10 & \multirow{2}{*}{$0.24 \pm 0.04$} & $0.19 \pm 0.00$ & $0.21 \pm 0.02$ & $0.08 \pm 0.00$ & $0.014 \pm 0.01$ \\
\hline & 1 & & $0.23 \pm 0.09$ & $0.24 \pm 0.027$ & $0.25 \pm 0$ & $0.03 \pm 0.0$ \\
\hline \multirow{2}{*}{ K. pneumonia (S) } & 10 & \multirow{2}{*}{$0.31 \pm 0.02$} & $0.22 \pm 0.03$ & $0.15 \pm 0.00$ & $0.10 \pm 0.06$ & $0.34 \pm 0.021$ \\
\hline & 1 & & $0.21 \pm 0.06$ & $0.26 \pm 0.0$ & $0.07 \pm 0.0$ & $0.21 \pm 0.024$ \\
\hline \multirow{2}{*}{ K. pneumonia (C) } & 10 & \multirow{2}{*}{$0.31 \pm 0.02$} & $0.23 \pm 0.03$ & $0.13 \pm 0.012$ & $0.35 \pm 0.025$ & $0.03 \pm 0.0$ \\
\hline & 1 & & $0.21 \pm 0.06$ & $0.13 \pm 0.010$ & $0.13 \pm 0.011$ & $0.018 \pm 0.0$ \\
\hline \multirow{2}{*}{ S. aureus $(\mathrm{S})$} & 10 & \multirow{2}{*}{$0.31 \pm 0.01$} & $0.22 \pm .03$ & $0.19 \pm 0.026$ & $0.14 \pm 0.09$ & $0.05 \pm 0.0$ \\
\hline & 1 & & $0.26 \pm 0.09$ & $0.27 \pm 0.021$ & $0.16 \pm 0.02$ & $0.21 \pm 0.02$ \\
\hline \multirow{2}{*}{ S. aureus (C) } & 10 & \multirow{2}{*}{$0.31 \pm 0.01$} & $0.125 \pm 0.0$ & $0.17 \pm 0.013$ & $0.13 \pm 0.010$ & $0.009 \pm 0.0$ \\
\hline & 1 & & $0.26 \pm 0.09$ & $0.17 \pm 0.013$ & $0.06 \pm 0.0$ & $0.03 \pm 0.0$ \\
\hline \multirow{2}{*}{ E. $\operatorname{coli}(\mathbf{S})$} & 10 & \multirow{2}{*}{$0.16 \pm 0.02$} & $0.11 \pm 0.00$ & $0.12 \pm 0.02$ & $0.29 \pm 0.19$ & $0.08 \pm 0.0$ \\
\hline & 1 & & $0.16 \pm 0.01$ & $0.19 \pm 0.026$ & $0.29 \pm 0.019$ & $0.02 \pm 0.0$ \\
\hline \multirow{2}{*}{ E. $\operatorname{coli}(\mathrm{C})$} & 10 & \multirow{2}{*}{$0.42 \pm 0.03$} & $0.14 \pm 0.00$ & $0.18 \pm 0.01$ & $0.081 \pm 0.0$ & $0.18 \pm 0.012$ \\
\hline & 1 & & $0.16 \pm 0.01$ & $0.27 \pm 0.022$ & $0.27 \pm 0.022$ & $0.37 \pm 0.021$ \\
\hline \multirow{2}{*}{ P. aeruginosa (S) } & 10 & \multirow{2}{*}{$0.43 \pm 0.05$} & $0.17 \pm 0.00$ & $0.18 \pm 0.00$ & $0.014 \pm 0.0$ & $0.0052 \pm 0.0$ \\
\hline & 1 & & $0.31 \pm 0.05$ & $0.15 \pm 0.003$ & $0.06 \pm 0.00$ & $0.05 \pm 0.0$ \\
\hline \multirow{2}{*}{ P. aeruginosa $(\mathrm{C})$} & 10 & \multirow{2}{*}{$0.43 \pm 0.05$} & $0.14 \pm 0.09$ & $0.27 \pm 0.02$ & $0.07 \pm 0.0$ & $0.072 \pm 0.0$ \\
\hline & 1 & & $0.31 \pm 0.05$ & $0.28 \pm 0.023$ & $0.24 \pm 0.05$ & $0.05 \pm 0.0$ \\
\hline \multirow{2}{*}{ S. typhi (S) } & 10 & \multirow{2}{*}{$0.16 \pm 0.01$} & $0.26 \pm 0.04$ & $0.11 \pm 0.024$ & $0.03 \pm 0.0$ & $0.13 \pm 0.01$ \\
\hline & 1 & & $0.13 \pm 0.04$ & $0.17 \pm 0.013$ & $0.04 \pm 0.0$ & $0.04 \pm 0.0$ \\
\hline \multirow{2}{*}{ S. typhi (C) } & 10 & \multirow{2}{*}{$0.16 \pm 0.01$} & $0.29 \pm 0.00$ & $0.16 \pm 0.00$ & $0.06 \pm 0.00$ & $0.10 \pm 0.012$ \\
\hline & 1 & & $0.13 \pm 0.04$ & $0.17 \pm 0.0$ & $0.11 \pm 0.04$ & $0.10 \pm 0.01$ \\
\hline \multicolumn{7}{|l|}{ Fungi } \\
\hline \multirow{2}{*}{ T. mentegrophytes (S) } & 10 & \multirow{2}{*}{$0.33 \pm 0.14$} & $0.25 \pm 0.21$ & $0.21 \pm 0.07$ & $0.23 \pm 0.23$ & $0.0625 \pm 0$ \\
\hline & 1 & & $0.33 \pm 0.14$ & $0.25 \pm 0.24$ & $0.33 \pm 0.14$ & $13 \pm 11.7$ \\
\hline \multirow{2}{*}{ C. neoformens (C) } & 10 & \multirow{2}{*}{$26.6 \pm 14.4$} & $11.5 \pm 11.8$ & $9.4 \pm 5.4$ & $18.7 \pm 7.2$ & $0.25 \pm 0.14$ \\
\hline & 1 & & $13.5 \pm 10.9$ & $14.5 \pm 9.5$ & $20.1 \pm 7.2$ & $16.7 \pm 10.8$ \\
\hline
\end{tabular}

doping. MIC values of T. mentegrophytes (s) and $C$. neoformens (c) were $0.21 \pm 0.07 \mathrm{mg} / \mathrm{ml}$ and $9.4 \pm 5.4$ $\mathrm{mg} / \mathrm{ml}$, respectively in case of $10 \% \mathrm{Mn}$ doping whereas $0.25 \pm 0.24 \mathrm{mg} / \mathrm{ml}$ and $14.5 \pm 9.5 \mathrm{mg} / \mathrm{ml}$ were obtained with $1 \% \mathrm{Mn}$ doping.

$10 \%$ cobalt $(\mathrm{Co})$ doped $\mathrm{ZnO}$ nanoparticle revealed a MIC range of $0.014-0.35 \mathrm{mg} / \mathrm{ml}$ with the most effective value at $0.03 \pm 0.0 \mathrm{mg} / \mathrm{ml}$ against S. typhi (s) closely followed by $0.06 \pm 0.0 \mathrm{mg} / \mathrm{ml}$ value against $S$. typhi (c). Again, S. typhi (s) gave superb MIC at a concentration of $0.04 \pm 0.0 \mathrm{mg} /$ $\mathrm{ml}$ with $1 \%$ Co doping trailed by $P$. aeruginosa (s) and $S$. aureus (s) at the value of $0.06 \pm 0.0 \mathrm{mg} /$ $\mathrm{ml}$ respectively. MIC values for T. mentegrophytes and $C$. neoformens were $0.23 \pm 0.23 \mathrm{mg} / \mathrm{ml}$ and $18.7 \pm 7.2 \mathrm{mg} / \mathrm{ml}$ respectively with $10 \%$ Co doping. MIC of T. mentegrophytes (s) against $1 \%$ doping was $0.33 \pm 0.14 \mathrm{mg} / \mathrm{ml}$ whereas for $C$. neoformens (c) was $20.1 \pm 7.2 \mathrm{mg} / \mathrm{ml}$ respectively.

An admirable minimum inhibitory concentration of $0.004 \pm 0 \mathrm{mg} / \mathrm{ml}$ against B. subtilis (s) was obtained with $10 \%$ copper $(\mathrm{Cu})$ doping which was 
better as compared to effective MIC of $1 \% \mathrm{Cu}$ doped $\mathrm{ZnO}$ nanoparticle against, $B$. subtilis (s) and K. pneumoniae (c) at the concentration of $0.018 \pm 0$ $\mathrm{mg} / \mathrm{ml}$. MIC values for both the fungal strains ( $T$. mentegrophytes (s) and C. neoformens (c)) were $0.0625 \pm 0 \mathrm{mg} / \mathrm{ml}$ and $13 \pm 11.7$ respectively in case of $10 \% \mathrm{Cu}$ doping and $0.25 \pm 0.14 \mathrm{mg} / \mathrm{ml}$ and $16.7 \pm 10.8 \mathrm{mg} / \mathrm{ml}$ respectively in case of $1 \% \mathrm{Cu}$ doping respectively.

MIC range of antimicrobials obtained isat: CIP: 0.5-4 $\mu \mathrm{g} / \mathrm{ml}$, Amp: 1-34 $\mu \mathrm{g} / \mathrm{ml}$, Flu: 0.12-16 $\mu \mathrm{g} / \mathrm{ml}$, Amp B: 0.015-2 $\mu \mathrm{g} / \mathrm{ml}$.

Synergistic Interaction (Table II-IV):

Interaction of nanoparticles with the antimicrobial agents was assessed by checkerboard as- say as summed up in tables II-IV. Synergistic and additive effects were found, no antagonistic effect was seen. The results are quite in terms with MIC clearly depicting that high doping agent is most effective antimicrobial agents. Though not much varied, but more synergistic effect was observed in when doped nanoparticles were combined with ciprofloxacin as compared to ampicillin. Undoped $\mathrm{ZnO}$ nanoparticles showed a synergistic effect when combined with ciprofloxacin against $K$. pneumoniae only. Additive effect was only observed for undoped $\mathrm{ZnO}$ nanoparticles when combined with ampicillin. Ciprofloxacin exhibited 100\% synergism (12/12 strains) for 10\% doped nanoparticles i.e. $10 \% \mathrm{Fe}, 10 \% \mathrm{Mn}, 10 \% \mathrm{Cu}$ and $10 \%$ Co doped $\mathrm{ZnO}$ nanoparticles. The least synergistic effect

TABLE II

Combined activity of nanoparticles with ciprofloxacin against different pathogenic microorganisms.

\begin{tabular}{|c|c|c|c|c|c|c|c|c|c|c|c|}
\hline \multirow[b]{2}{*}{ Microorganisms } & \multicolumn{11}{|c|}{ FIC Value } \\
\hline & $\begin{array}{c}\text { Doping } \\
\%\end{array}$ & Fe doping & $\begin{array}{l}\text { S/ } \\
\text { A/ } \\
\text { An }\end{array}$ & Mn doping & $\begin{array}{l}\text { S/ } \\
\text { A/ } \\
\text { An }\end{array}$ & Co doping & $\begin{array}{l}\text { S/ } \\
\text { A/ } \\
\text { An }\end{array}$ & Cu doping & $\begin{array}{l}\text { S/ } \\
\text { A/ } \\
\text { An }\end{array}$ & Pure ZnO & $\begin{array}{l}\text { S/ } \\
\text { A/ } \\
\text { An }\end{array}$ \\
\hline \multirow{2}{*}{ B. subtilis (s) } & 10 & $0.24 \pm 0.08$ & $\mathrm{~S}$ & $0.17 \pm 0.10$ & $\mathrm{~S}$ & $0.40 \pm 0.07$ & $\mathrm{~S}$ & $0.41 \pm 0.05$ & $\mathrm{~S}$ & \multirow{2}{*}{$0.61 \pm 0.02$} & \multirow{2}{*}{ A } \\
\hline & 1 & $0.46 \pm 0.08$ & $\mathrm{~S}$ & $0.36 \pm 0.05$ & $\mathrm{~S}$ & $0.33 \pm 0.06$ & $\mathrm{~S}$ & $0.45 \pm 0.06$ & $\mathrm{~S}$ & & \\
\hline \multirow{2}{*}{ B. subtilis (C) } & 10 & $0.35 \pm 0.06$ & $\mathrm{~S}$ & $0.21 \pm 0.08$ & $\mathrm{~S}$ & $0.45 \pm 0.05$ & $\mathrm{~S}$ & $0.41 \pm 0.07$ & $\mathrm{~S}$ & \multirow{2}{*}{$0.52 \pm 0.05$} & \multirow{2}{*}{ A } \\
\hline & 1 & $0.48 \pm 0.05$ & $\mathrm{~S}$ & $0.54 \pm 0.06$ & A & $0.54 \pm 0.06$ & A & $0.46 \pm 0.07$ & $\mathrm{~S}$ & & \\
\hline \multirow{2}{*}{ K. pneumonia (S) } & 10 & $0.26 \pm 0.05$ & $\mathrm{~S}$ & $0.25 \pm 0.06$ & $\mathrm{~S}$ & $0.25 \pm 0.10$ & $\mathrm{~S}$ & $0.27 \pm 0.04$ & $\mathrm{~S}$ & \multirow{2}{*}{$0.46 \pm 0.10$} & \multirow{2}{*}{$\mathrm{S}$} \\
\hline & 1 & $0.57 \pm 0.07$ & A & $0.42 \pm 0.08$ & $\mathrm{~S}$ & $0.46 \pm 0.11$ & $\mathrm{~S}$ & $0.4 \pm 0.12$ & $\mathrm{~S}$ & & \\
\hline \multirow{2}{*}{ K. pneumonia $(\mathrm{C})$} & 10 & $0.26 \pm 0.09$ & $\mathrm{~S}$ & $0.22 \pm 0.11$ & $\mathrm{~S}$ & $0.20 \pm 0.14$ & $\mathrm{~S}$ & $0.20 \pm 0.16$ & $\mathrm{~S}$ & \multirow{2}{*}{$0.33 \pm 0.18$} & \multirow{2}{*}{$\mathrm{S}$} \\
\hline & 1 & $0.22 \pm 0.18$ & $\mathrm{~S}$ & $0.30 \pm 0.16$ & $\mathrm{~S}$ & $0.27 \pm 0.12$ & $\mathrm{~S}$ & $0.27 \pm 0.03$ & $\mathrm{~S}$ & & \\
\hline \multirow{2}{*}{ S. aureus (S) } & 10 & $0.34 \pm 0.02$ & $\mathrm{~S}$ & $0.36 \pm 0.03$ & $\mathrm{~S}$ & $0.24 \pm 0.08$ & $\mathrm{~S}$ & $0.39 \pm 0.05$ & $\mathrm{~S}$ & \multirow{2}{*}{$0.69 \pm 0.16$} & \multirow{2}{*}{ A } \\
\hline & 1 & $0.48 \pm 0.31$ & $\mathrm{~S}$ & $0.27 \pm 0.08$ & $\mathrm{~S}$ & $0.34 \pm 0.15$ & $\mathrm{~S}$ & $0.47 \pm 0.17$ & $\mathrm{~S}$ & & \\
\hline \multirow{2}{*}{ S. aureus $(\mathrm{C})$} & 10 & $0.33 \pm 0.08$ & $\mathrm{~S}$ & $0.38 \pm 0.09$ & $\mathrm{~S}$ & $0.37 \pm 0.07$ & $\mathrm{~S}$ & $0.48 \pm 0.12$ & $\mathrm{~S}$ & \multirow{2}{*}{$0.62 \pm 0.21$} & \multirow{2}{*}{ A } \\
\hline & 1 & $0.64 \pm 0.29$ & A & $0.79 \pm 0.09$ & A & $0.45 \pm 0.06$ & $\mathrm{~S}$ & $0.84 \pm 0.14$ & A & & \\
\hline \multirow{2}{*}{ E. $\operatorname{coli}(\mathrm{S})$} & 10 & $0.39 \pm 0.14$ & $\mathrm{~S}$ & $0.44 \pm 0.15$ & $\mathrm{~S}$ & $0.31 \pm 0.14$ & $\mathrm{~S}$ & $0.17 \pm 0.10$ & $\mathrm{~S}$ & \multirow{2}{*}{$0.74 \pm 0.15$} & \multirow{2}{*}{ A } \\
\hline & 1 & $0.44 \pm 0.12$ & $\mathrm{~S}$ & $0.46 \pm 0.04$ & $\mathrm{~S}$ & $0.62 \pm 0.13$ & A & $0.61 \pm 0.19$ & A & & \\
\hline \multirow{2}{*}{ E. coli $(\mathrm{C})$} & 10 & $0.48 \pm 0.19$ & $\mathrm{~S}$ & $0.43 \pm 0.16$ & $\mathrm{~S}$ & $0.46 \pm 0.22$ & $\mathrm{~S}$ & $0.33 \pm 0.19$ & $\mathrm{~S}$ & \multirow{2}{*}{$0.69 \pm 0.06$} & \multirow{2}{*}{ A } \\
\hline & 1 & $0.47 \pm 0.16$ & $\mathrm{~S}$ & $0.82 \pm 0.16$ & $\mathrm{~A}$ & $0.49 \pm 0.08$ & $\mathrm{~S}$ & $0.72 \pm 0.08$ & $\mathrm{~A}$ & & \\
\hline \multirow{2}{*}{ P. aeruginosa $(\mathrm{S})$} & 10 & $0.42 \pm 0.08$ & $\mathrm{~S}$ & $0.32 \pm 0.12$ & $\mathrm{~S}$ & $0.2 \pm 0.03$ & $\mathrm{~S}$ & $0.39 \pm 0.06$ & $\mathrm{~S}$ & \multirow{2}{*}{$0.54 \pm 0.09$} & \multirow{2}{*}{ A } \\
\hline & 1 & $0.46 \pm 0.09$ & $\mathrm{~S}$ & $0.24 \pm 0.08$ & $\mathrm{~S}$ & $0.36 \pm 0.17$ & $\mathrm{~S}$ & $0.32 \pm 0.04$ & $\mathrm{~S}$ & & \\
\hline \multirow{2}{*}{ P. aeruginosa (C) } & 10 & $0.25 \pm 0.16$ & $\mathrm{~S}$ & $0.43 \pm 0.05$ & $\mathrm{~S}$ & $0.21 \pm 0.06$ & $\mathrm{~S}$ & $0.36 \pm 0.08$ & $\mathrm{~S}$ & $094+007$ & $A$ \\
\hline & 1 & $0.38 \pm 0.09$ & $\mathrm{~S}$ & $0.83 \pm 0.11$ & $\mathrm{~A}$ & $0.21 \pm 0.06$ & $\mathrm{~S}$ & $0.46 \pm 0.05$ & $\mathrm{~S}$ & $0.94 \pm 0.01$ & A \\
\hline S & 10 & $0.33 \pm 0.11$ & $\mathrm{~S}$ & $0.38 \pm 0.12$ & $\mathrm{~S}$ & $0.28 \pm 0.06$ & $\mathrm{~S}$ & $0.26 \pm 0.09$ & $\mathrm{~S}$ & & $\mathrm{~A}$ \\
\hline ग. Чурп (s) & 1 & $0.7 \pm 0.25$ & A & $0.47 \pm 0.08$ & $\mathrm{~S}$ & $0.45 \pm 0.08$ & $\mathrm{~S}$ & $0.34 \pm 0.04$ & $\mathrm{~S}$ & $0.02 \pm 0.15$ & A \\
\hline & 10 & $0.29 \pm 0.03$ & $\mathrm{~S}$ & $0.45 \pm 0.05$ & $\mathrm{~S}$ & $0.29 \pm 0.14$ & $\mathrm{~S}$ & $0.35 \pm 0.08$ & $\mathrm{~S}$ & & A \\
\hline ग. уури (с) & 1 & $0.36 \pm 0.06$ & $\mathrm{~S}$ & $0.68 \pm 0.17$ & A & $0.48 \pm 0.02$ & $\mathrm{~S}$ & $0.77 \pm 0.07$ & A & $0.02 \pm 0.03$ & A \\
\hline
\end{tabular}


TABLE III

Combined activity of nanoparticles with ampicillin against different pathogenic microorganisms.

\begin{tabular}{|c|c|c|c|c|c|c|c|c|c|c|c|}
\hline & \multicolumn{11}{|c|}{ FIC Value } \\
\hline Microorganisms & $\begin{array}{c}\text { Doping } \\
\%\end{array}$ & Fe doping & $\begin{array}{c}\text { S/ } \\
\text { A/ } \\
\text { An }\end{array}$ & Mn doping & $\begin{array}{l}\text { S/ } \\
\text { A/ } \\
\text { An }\end{array}$ & Co doping & $\begin{array}{l}\text { S/ } \\
\text { A/ } \\
\text { An }\end{array}$ & $\mathrm{Cu}$ doping & $\begin{array}{l}\text { S/ } \\
\text { A/ } \\
\text { An }\end{array}$ & Pure ZnO & $\begin{array}{l}\text { S/ } \\
\text { A/ } \\
\text { An }\end{array}$ \\
\hline \multirow{2}{*}{ B. subtilis (s) } & 10 & $0.27 \pm 0.02$ & $\mathrm{~S}$ & $0.23 \pm 0.04$ & $\mathrm{~S}$ & $0.35 \pm 0.07$ & $\mathrm{~S}$ & $0.37 \pm 0.09$ & $\mathrm{~S}$ & \multirow{2}{*}{$0.57 \pm 0.04$} & \multirow{2}{*}{ A } \\
\hline & 1 & $0.44 \pm 0.09$ & $\mathrm{~S}$ & $0.39 \pm 0.05$ & $\mathrm{~S}$ & $0.51 \pm 0.02$ & A & $0.41 \pm 0.02$ & S & & \\
\hline \multirow{2}{*}{ B. subtilis (C) } & 10 & $0.39 \pm 0.03$ & $\mathrm{~S}$ & $0.39 \pm 0.05$ & $\mathrm{~S}$ & $0.44 \pm 0.10$ & $\mathrm{~S}$ & $0.42 \pm 0.04$ & $\mathrm{~S}$ & \multirow{2}{*}{$0.77 \pm 0.03$} & \multirow{2}{*}{ A } \\
\hline & 1 & $0.46 \pm 0.06$ & $\mathrm{~S}$ & $0.44 \pm 0.04$ & $\mathrm{~S}$ & $0.52 \pm 0.01$ & A & $0.49 \pm 0.02$ & $\mathrm{~S}$ & & \\
\hline \multirow{2}{*}{ K. pneumonia (S) } & 10 & $0.26 \pm 0.11$ & $\mathrm{~S}$ & $0.3 \pm 0.02$ & $\mathrm{~S}$ & $0.24 \pm 0.05$ & $\mathrm{~S}$ & $0.39 \pm 0.02$ & $\mathrm{~S}$ & \multirow{2}{*}{$0.67 \pm 0.09$} & \multirow{2}{*}{ A } \\
\hline & 1 & $0.48 \pm 0.04$ & $\mathrm{~S}$ & $0.42 \pm 0.0$ & $\mathrm{~S}$ & $0.43 \pm 0.08$ & $\mathrm{~S}$ & $0.52 \pm 0.04$ & A & & \\
\hline \multirow{2}{*}{ K. pneumonia $(\mathrm{C})$} & 10 & $0.32 \pm 0.05$ & $\mathrm{~S}$ & $0.34 \pm 0.07$ & $\mathrm{~S}$ & $0.36 \pm 0.11$ & $\mathrm{~S}$ & $0.48 \pm 0.02$ & $\mathrm{~S}$ & \multirow{2}{*}{$0.6 \pm 0.13$} & \multirow{2}{*}{ A } \\
\hline & 1 & $0.51 \pm 0.02$ & A & $0.42 \pm 0.0$ & $\mathrm{~S}$ & $0.53 \pm 0.01$ & A & $0.61 \pm 0.12$ & A & & \\
\hline \multirow{2}{*}{ S. aureus (S) } & 10 & $0.40 \pm 0.08$ & $\mathrm{~S}$ & $0.38 \pm 0.07$ & $\mathrm{~S}$ & $0.25 \pm 0.06$ & $\mathrm{~S}$ & $0.36 \pm 0.08$ & $\mathrm{~S}$ & \multirow{2}{*}{$0.58 \pm 0.05$} & \multirow{2}{*}{ A } \\
\hline & 1 & $0.43 \pm 0.05$ & $\mathrm{~S}$ & $0.49 \pm 0.06$ & $\mathrm{~S}$ & $0.45 \pm 0.06$ & $\mathrm{~S}$ & $0.57 \pm 0.09$ & A & & \\
\hline \multirow{2}{*}{ S. aureus (C) } & 10 & $0.41 \pm 0.06$ & $\mathrm{~S}$ & $0.31 \pm 0.02$ & $\mathrm{~S}$ & $0.24 \pm 0.13$ & $\mathrm{~S}$ & $0.44 \pm 0.07$ & $\mathrm{~S}$ & \multirow{2}{*}{$0.69 \pm 0.05$} & \multirow{2}{*}{ A } \\
\hline & 1 & $0.46 \pm 0.0$ & $\mathrm{~S}$ & $0.48 \pm 0.02$ & $\mathrm{~S}$ & $0.47 \pm 0.09$ & $\mathrm{~S}$ & $0.57 \pm 0.05$ & A & & \\
\hline \multirow{2}{*}{ E. $\operatorname{coli}(\mathbf{S})$} & 10 & $0.33 \pm 0.07$ & $\mathrm{~S}$ & $0.25 \pm 0.07$ & $\mathrm{~S}$ & $0.39 \pm 0.08$ & $\mathrm{~S}$ & $0.38 \pm 0.04$ & $\mathrm{~S}$ & \multirow{2}{*}{$0.64 \pm 0.07$} & \multirow{2}{*}{ A } \\
\hline & 1 & $0.43 \pm 0.07$ & $\mathrm{~S}$ & $0.56 \pm 0.05$ & A & $0.47 \pm 0.03$ & $\mathrm{~S}$ & $0.43 \pm 0.03$ & $\mathrm{~S}$ & & \\
\hline \multirow{2}{*}{ E. $\operatorname{coli}(\mathrm{C})$} & 10 & $0.47 \pm 0.03$ & $\mathrm{~S}$ & $0.41 \pm 0.03$ & $\mathrm{~S}$ & $0.49 \pm 0.03$ & $\mathrm{~S}$ & $0.46 \pm 0.03$ & $\mathrm{~S}$ & \multirow{2}{*}{$0.66 \pm 0.07$} & \multirow{2}{*}{ A } \\
\hline & 1 & $0.52 \pm 0.03$ & A & $0.5 \pm 0.01$ & $\mathrm{~S}$ & $0.55 \pm 0.07$ & A & $0.52 \pm 0.04$ & A & & \\
\hline \multirow{2}{*}{ P. aeruginosa $(\mathrm{S})$} & 10 & $0.27 \pm 0.03$ & $\mathrm{~S}$ & $0.21 \pm 0.05$ & $\mathrm{~S}$ & $0.36 \pm 0.05$ & $\mathrm{~S}$ & $0.40 \pm .03$ & $\mathrm{~S}$ & \multirow{2}{*}{$0.64 \pm 0.09$} & \multirow{2}{*}{ A } \\
\hline & 1 & $0.33 \pm 0.11$ & $\mathrm{~S}$ & $0.33 \pm 0.11$ & $\mathrm{~S}$ & $0.43 \pm 0.09$ & $\mathrm{~S}$ & $0.52 \pm 0.06$ & A & & \\
\hline \multirow{2}{*}{ P. aeruginosa $(\mathrm{C})$} & 10 & $0.44 \pm 0.11$ & $\mathrm{~S}$ & $0.39 \pm 0.02$ & $\mathrm{~S}$ & $0.45 \pm 0.15$ & $\mathrm{~S}$ & $0.36 \pm 0.06$ & $\mathrm{~S}$ & $060+003$ & A \\
\hline & 1 & $0.49 \pm 0.02$ & $\mathrm{~S}$ & $0.44 \pm 0.07$ & $\mathrm{~S}$ & $0.63 \pm 0.08$ & A & $0.5 \pm 0.03$ & $\mathrm{~S}$ & $0.00+0.00$ & 11 \\
\hline S tonhi(S) & 10 & $0.32 \pm 0.04$ & $\mathrm{~S}$ & $0.27 \pm 0.09$ & $\mathrm{~S}$ & $0.3 \pm 0.09$ & $\mathrm{~S}$ & $0.38 \pm 0.04$ & $\mathrm{~S}$ & $051+005$ & $A$ \\
\hline 2. & 1 & $0.43 \pm 0.08$ & $\mathrm{~S}$ & $0.33 \pm 0.02$ & $\mathrm{~S}$ & $0.44 \pm 0.08$ & $\mathrm{~S}$ & $0.47 \pm 0.03$ & $\mathrm{~S}$ & $0.01+0.00$ & $n$ \\
\hline & 10 & $0.45 \pm 0.04$ & $\mathrm{~S}$ & $0.33 \pm 0.06$ & $\mathrm{~S}$ & $0.32 \pm 0.04$ & $\mathrm{~S}$ & $0.40 \pm 0.02$ & $\mathrm{~S}$ & $055+008$ & \\
\hline נ) (2) & 1 & $0.55 \pm 0.04$ & A & $0.46 \pm 0.03$ & $\mathrm{~S}$ & $0.50 \pm 0.09$ & $\mathrm{~S}$ & $0.53 \pm 0.07$ & A & . & 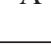 \\
\hline
\end{tabular}

TABLE IV

Combined activity of nanoparticles with fluconazole and amphotericin $B$ against fungi.

\begin{tabular}{lcccc}
\hline Nanoparticles & C.neoformens + Flu & S/A/An & T. mentegrophyte + Amp B & S/A/An \\
\hline $\mathbf{Z n O}$ & $0.68 \pm 0.01$ & $\mathrm{~A}$ & $0.50 \pm 0.09$ & $\mathrm{~A}$ \\
\hline $\mathbf{Z n O + 1 0 \% F e}$ & $0.51 \pm 0.02$ & $\mathrm{~A}$ & $0.47 \pm 0.02$ & $\mathrm{~A}$ \\
\hline $\mathbf{Z n O + 1 \% F e}$ & $0.63 \pm 0.05$ & $\mathrm{~A}$ & $0.52 \pm 0.02$ & $\mathrm{~A}$ \\
\hline $\mathbf{Z n O + 1 0 \% M n}$ & $0.5 \pm 0.01$ & $\mathrm{~A}$ & $0.3 \pm 0.11$ & $\mathrm{~S}$ \\
\hline $\mathbf{Z n O + 1 \% M n}$ & $0.54 \pm 0.03$ & $\mathrm{~A}$ & $0.41 \pm 0.04$ & $\mathrm{~S}$ \\
\hline $\mathbf{Z n O + 1 0 \% C o}$ & $0.56 \pm 0.03$ & $\mathrm{~A}$ & $0.3 \pm 0.01$ & $\mathrm{~S}$ \\
\hline $\mathbf{Z n O + 1 \% C o}$ & $0.64 \pm 0.03$ & $\mathrm{~A}$ & $0.4 \pm 0.03$ & $\mathrm{~S}$ \\
\hline $\mathbf{Z n O + 1 0 \% C u}$ & $0.46 \pm 0.03$ & $\mathrm{~A}$ & $0.34 \pm 0.07$ & $\mathrm{~S}$ \\
\hline $\mathbf{Z n O + 1 \% C u}$ & $0.74 \pm 0.05$ & $\mathrm{~A}$ & $0.49 \pm 0.05$ & $\mathrm{~A}$ \\
\hline
\end{tabular}


was shown by $1 \% \mathrm{Mn}$ doped $\mathrm{ZnO}$ nanoparticles i.e. only $58.3 \%$ (7/12). Interaction of ciprofloxacin with $10 \% \mathrm{Mn}$ and $10 \% \mathrm{Cu}$ revealed strongest synergistic effect on the FIC value of 0.17 against both B. subtilis (s) and E. coli (s). For ampicillin, also $10 \%$ doped nanoparticles presented $100 \%$ synergistic activity. Only 41.6\% (5/12) synergism was shown by $1 \% \mathrm{Cu}$ doped $\mathrm{ZnO}$ nanoparticles, which was least among all the doped nanoparticles used in the study. Further, in case of ampicillin, 10\% Mn with ampicillin showed sturdiest synergistic effect against $P$. aeruginosa (s) at FIC value of 0.21 .

Mostly additive effect was shown by the fungi (Table IV). $100 \%$ additive effect was shown by the combination of $C$. neoformens and fluconazole. Except $10 \%$ and $1 \% \mathrm{Mn}$ doped, $10 \%$ and $1 \%$ Co doped and $10 \% \mathrm{Cu}$ doped $\mathrm{ZnO}$ nanoparticles all other exhibited the additive effect for $T$. mentegrophytes and amphotericin B combination. Of these most promising synergistic effect was shown by $10 \%$ Co at FIC value of $0.3 \pm 0.01$.

\section{DISCUSSION}

The issue of resistance to antibiotics and its diffusion, however, are major health troubles, leading to treatment drawbacks for a large number of drugs (Braga et al. 2005, Schito 2006). Drug resistance enforces high dose administration of antibiotics, often generating intolerable toxicity, development of new antibiotics, and requests for significant economic, labor, and time investments. It has been assumed that if present trends continue, antibiotic failure will claim 10 million lives per year by 2050 (O'Neill 2014). Therefore, there has been increasing interest in the role of inhibitors of antibiotic resistance for combination therapy (Gibbons 2005, Wright 2005). Nanotechnology represents a modern and forward-looking approach to develop new formulations based upon metallic nanoparticles with antimicrobial properties. Therapeutic roles for zinc in different diseases have been demonstrated in recent years.
With the goal of developing a new highly active antimicrobial therapeutic combination of nanoparticles and synthetic antimicrobial agents, we began by comparing the antimicrobial properties of nine nanoparticles against different pathogenic bacterial and fungal strains. Higher doped nanoparticles $(10 \%)$ revealed better antimicrobial property than the lesser doped (1\%) and undoped. The MIC against test strains shows that nanoparticles have a less significant effect on growth of gram-positive bacteria than on gram-negative bacteria. This is due to the structural difference in cell wall composition of gram-positive and gramnegative bacteria. The gram-negative bacteria have a layer of lipopolysaccharides at the exterior, followed underneath by a thin $(\sim 7-8 \mathrm{~nm})$ layer of peptidoglycan (Madigan and Martinko 2005). Although the lipopolysaccharides are composed of covalently linked lipids and polysaccharides, there is a lack of strength and rigidity. The negative charges on lipopolysaccharides are attracted toward the weak positive charge available on the doped and undoped $\mathrm{ZnO}$ nanoparticles (Sui et al. 2006). On the other hand, the cell wall in gram-positive bacteria are principally composed of a thick layer ( 20-80 $\mathrm{nm}$ ) of peptidoglycan consisting of linear polysaccharide chains cross-linked by short peptides to form a three-dimensional rigid structure (Baron 1996). The rigidity and extended cross-linking not only endow the cell walls with fewer anchoring sites for the doped and undoped $\mathrm{ZnO}$ nanoparticles but also make them difficult to penetrate.

Regards to mycoses, nanoparticles can be considered as a potential antifungal agent. Our results showed better antifungal activity of $\mathrm{ZnO}$ nanoparticles against T. mentagrophytes, as compared to earlier studies of Sawai and Yoshikawa (2004). The antifungal effect of doped and undoped $\mathrm{ZnO}$ nanoparticles particularly the doped has received only marginal attention and just a few studies on this topic has been published. Our study suggests 
the better antifungal activity of doped nanoparticles than pure nanoparticles. Thereby, further supporting the fact that doping increases antimicrobial activity.

There is a significant difference in the MIC values of undoped $\mathrm{ZnO}$ nanoparticles and doped $\mathrm{ZnO}$ nanoparticles. This indicates the fact that dopant was interfering with the active principle as the MIC values are greater in doped $\mathrm{ZnO}$ nanoparticles as compared to undoped $\mathrm{ZnO}$ nanoparticles for different groups of bacteria and fungi. It has been found from some previous studies that doping may increase the antimicrobial effect (Rekha et al. 2010). Dopant impurities like $\mathrm{Cu}^{2+}$, $\mathrm{Mn}^{2+}, \mathrm{Co}^{2+}, \mathrm{Ni}^{2+}$, rare earth and transition elements, brings about significant changes in the physical, chemical and biological properties of host material on doping depending on the type of dopant and its concentration (Peng et al. 2006, Zhang et al. 2006). The increase in antibacterial and photocatalytic activity with increase in dopants concentration is attributed to the generation of reactive oxygen species (ROS).

For many years, antimicrobial drugs have been used to inhibit or kill bacteria and other microbes. However, microbial resistance to these drugs has developed on a very large scale over time, greatly reducing their effectiveness, and is an ever growing problem (Hajipour et al. 2012). One of the most promising strategies for overcoming microbial resistance is the use of nanoparticles. Development of resistance to these nanoparticles is, again, unlikely (Friedman et al. 2013), possibly because it would require multiple simultaneous gene mutations in the same microbial cell. Therefore, the interaction of nanoparticles with commonly used antimicrobials was studied by checkerboard method. The checkerboard test measures the inhibitory concentration. Here also results revealed better activity of doped nanoparticles than the undoped. Further strengthening the fact that doping increase the antimicrobial activity. Recent studies have shown that combining nanoparticles with antibiotics not only reduces the toxicity of both the agents towards human cells by decreasing their requirement or high dosages but also enhances their antimicrobial properties. Combining antibiotics with nanoparticles also restores their ability to destroy microbes that have acquired resistance to them. Furthermore, nanoparticles tagged with antibiotics have been shown to increase the concentration of the antibiotics at the site of bacterium-antibiotic interaction, and facilitate in the binding of antibiotics to microorganisms (Allahverdiyev et al. 2011). Our results are in terms with the study conducted by Banoee et al. (2010) showing better activity of ciprofloxacin nanoparticle combination as compared to ampicillin nanoparticle combination. They reported 27 and $22 \%$ increase in inhibition zone areas was observed against $S$. aureus and $E$. coli, respectively when $\mathrm{ZnO}$ nanoparticles combine with ciprofloxacin. However, our study is contradictory to the study conducted by Gaddad et al. (2010) who reported good activity of ampicillin and moderate activity of ciprofloxacin against $S$. aureus. Increased activity of ciprofloxacin in presence of doped $\mathrm{ZnO}$ nanoparticles can be attributed to inhibition of ciprofloxacin efflux from the cell due to interference of the doped $\mathrm{ZnO}$ nanoparticles with pumping NorA protein, activation of ciprofloxacin uptake by influencing activity of membrane Omf protein, and by binding reaction between ciprofloxacin and $\mathrm{ZnO}$ nanoparticles stabilizing the ciprofloxacindoped $\mathrm{ZnO}$ nanoparticle complex. This is done by the presence of fluore group and carboxyl group on the ciprofloxacin. The fluore group interacts with the chelating $\mathrm{Zn}$ atom stabilizing the ciprofloxacinnanoparticle combination whereas carboxyl group serves as an obvious target for chelation by metal ions (Banoee et al. 2010). Moreover, presence of doping ion on $\mathrm{ZnO}$ nanoparticle surface accelerates generation of reactive oxygen species (ROS) due to coupling or synergistic effects of $\mathrm{Mn}$, 
$\mathrm{Co}, \mathrm{Cu}$ and $\mathrm{Fe}$ loading. Further with an increase in concentration of doping synergistic activity increased as interaction between oxygen and dehydrogenase enzyme increases which increases the activity (Sikong et al. 2010). Minimal enhanced or unchanged antimicrobial activity of the other tested antibiotics against bacteria and funguscan be elucidated either by formation of weak hydrogen bonds with hydroxylated doped and undoped $\mathrm{ZnO}$ nanoparticles or by lack of sufficient targets for interaction (Banoee et al. 2010).

\section{ACKNOWLEDGMENTS}

The authors are grateful to Shoolini University, Department of Biotechnology, Bajhol, Solan, Himachal Pradesh, India for support and institutional facilities.

\section{RESUMO}

A combinação de antibióticos e nanoparticulas podem ser usadas para tratamento terapêutico de microoganismos multi-resistentes. Nanoparticulas (NPs) tem sido relatada com atividade antimicrobial. A atividade antimicrobial de nanopartículas de $\mathrm{ZnO}$ dopadas ( $\mathrm{ZnO} \mathrm{NPs}$ ) foram estudas contra fungos, e bactérias gram-postivas e gram-negativas, usando o método de microdiluição. A interação entre as nanoparticulas e antibioticos foi estimada por cálculos de Concentração Inibitória Fracionada (FIC índice) da combinação através do ensaio do Tabuleiro de Xadrez. Os resultados demonstraram que as nanopartículas de Oxido de Zinco dopadas 10\% (ZnO NPs) exibem o máximo efeito antimicrobiano em contraste com aquelas carregadas em $1 \%$ e as puras $\mathrm{ZnO}$ nanoparticulas. O aumento do efeito antimicrobiano foi observado quando combinadas com antibióticos. Efeitos sinérgicos e aditivos foram observados. Não foram observados efeitos de antagonismo. Maior efeito sinérgico foi observado quando combinado com ciprofloxania e ampicilina. Fungos apresentaram somente efeito aditivo. Os resultados estão de acordo com MIC mostrando claramente que agentes com alta dopagem são mais propícios para terapia combinada. $100 \%$ de interação sinergística foi observada na maior dopagem com ciprofloxacina e ampicilina. Este estudo fornece um relato preliminar da atividade sinergística de nanopartículas com antibióticos contra diferentes linhagens patogênicas. Isto fornece base para novos estudos em relação a terapia combinada de nanopartículas com antibióticos.

Palavras-chave: atividade antimicrobiana, MIC, nanopartículas de $\mathrm{ZnO}$, FIC, ensaio de tabuleiro de xadrez.

\section{REFERENCES}

ALLAHVERDiYeV AM, KON KV, ABAMOR ES, BAGIROVA M AND RAFAILOVICH M. 2011. Coping with antibiotic resistance: combining nanoparticles with antibiotics and other antimicrobial agents. Expert Rev Anti Infect Ther 9(11): 1035-1052.

BANOEE M, SEIF S, JAFARI-FESHARAKI P, SHAHVERDI HR, Moballegh A, MOghaddam KM AND SHAHVERd AR. 2010. ZnO nanoparticles enhanced antibacterial activity of ciprofloxacin against Staphylococcus aureus and Escherichia coli. J Biomed Mater Res B Appl Biomater 93(2): 557-561.

BARON S. 1996. Structure (Salton MRJ, Kim KS). Medical microbiology. $4^{\text {th }}$ ed., Galveston: University of Texas Medical Branch, Chapter 2: 1-19.

BRAGA LC, LEITE AAM, XAVIER KGS, TAKAHASHI JA, BEMQUERER MP, CHARTONE-SOUZA E AND NASCIMENTO AMA. 2005. Synergic interaction between pomegranate extract and antibiotics against Staphylococcus aureus. Can J Microbiol 51: 541-547.

CLSI - CHEMICAL LABORATORY STANDARDS INSTITUTE. 2006. Methods for Dilution Antimicrobial susceptibility tests for bacteria that grow aerobically; Approved standard - seventh addition. CLSI document M7- A7 (ISBNI -56238 - 587- 9). CLSI, Wayne, Pennsylvania 19087- 1898, USA.

CLSI - CHEMICAL LABORATORY STANDARDS INSTITUTE. 2008a. Reference method for Broth Dillution Antifungal susceptibility testing of filamentous fungi; Approved standard-second edition. CLSI document M38-A2. CLSI, Wayne, Pennsylvania 19087-1898, USA.

CLSI - CHEMICAL LABORATORY STANDARDS INSTITUTE. 2008b. Reference method for broth dilution antifungal susceptibility testing of yeasts; approved standard- Third Edition. CLSI document M 27- A3 (ISBN -1-56238-6662). CLSI Wayne, Pennsylvania 19087-1898, USA.

Edmond MB, WALlace SE, MCClish DK, PFALler MA, JONES RN AND WENZEL RP. 1999. Nosocomial blood stream infections in United States hospitals: a 3-year analysis. J Clin Infect Dis 29: 239-244.

Eliopoulos GM AND MOELlering Jr RC. 1991. Laboratory methods used to assess the activity of antimicrobial combinations. In Antibiotics in Laboratory 
Medicine, $3^{\text {rd }}$ ed., Edited by V. Lorian. Baltimore, MD: Williams \& Wilkins, p. 432-492.

FRIEDMAN AJ ET AL. 2013. Antimicrobial and antiinflammatory activity of chitosan-alginate nanoparticles: a targeted therapy for cutaneous pathogens. J Investig Dermatol 133: 1231-1239.

GADDAD SM, THATI V, ROY AS, AMBIKA PRASAD MVN AND SHIVANNAVAR CT. 2010. Nanostructured Zinc Oxide Enhances the Activity of Antibiotics against Staphylococcus aureus. J Biosci Technol 1: 64-69.

GIBBONS S. 2005. Plants as a source of bacterial resistance modulators and anti-infective agents. Phytochem Rev 4: 63-78.

HAJIPOUR MJ, FROMM KM, AKBARASHKARRAN A, JIMENEZ DE ABERASTURI D, LARRAMENDI IRD, ROJO T, SERpooshan V, PARAK WJ AND MAHMOUdi M. 2012. Antibacterial properties of nanoparticles. Trends 30(10): 499-511.

HAMMER KA, CARSON CF AND RILEY TV. 1998. Invitro activity of essential oils, in particular Melaleuca alternifolia (tea tree) oil and tea tree oil products, against Candida spp. J Antimicrob Chemoth 42: 591-595.

KURODA M ET AL. 2001. Whole genome sequencing of methicillin-resistant Staphylococcus aureus. The Lancet 357: 1225-1240.

MADIGAN M AND MARTINKO J. 2005. Brock biology of microorganisms. $11^{\text {th }}$ ed., Englewood Cliffs, NJ: Prentice Hall, p. 1-34.

ODDS FC. 2003. Synergy, antagonism and what the chequerboard puts between them. J Antimicrob Chemother 52: 1 .

O’NeILl J. 2014. Review on Antimicrobial Resistance. Antimicrobial Resistance: Tackling a Crisis for the Health and Wealth of Nations. Supported by UK government and Wellcome Trust.

PENG WQ, CONG GW, QU SC AND WANG ZG. 2006. Synthesis and photoluminescence of $\mathrm{ZnS}$ : Cu nanoparticles. Opt Mat 29: 313-317.

Pillai SK, Moellering Jr RC AND Eliopoulos GM. 2005. Antimicrobial combinations. In: Lorian V (Ed), Antibiotics in laboratory medicine, $5^{\text {th }}$ ed., Lippincott Williams \& Wilkins, Philadelphia, PA, p. 365-440

RANi A, JAIN S, DUREJA P, Kumar R AND Kumar A. 2009. Synergistic interaction between synthetic and natural products: a promising tool for the development of environmentally safe potent antimicrobial agents. World Appl Sci J 5(Special Issue for Environment): 59-63.

REKHA K, NiRMALA M, NAIR MG AND ANUKALIANI A. 2010. Structural, optical, photocatalytic and antibacterial activity of zinc oxide and manganese doped zinc oxide nanoparticles. Physica B: Condensed Matter 405(15): 3180-3185.

SAWAI J AND YOSHIKAWA T. 2004. Quantitative evaluation of antifungal activity of metallic oxide powders $(\mathrm{MgO}$, $\mathrm{CaO}$ and $\mathrm{ZnO}$ ) by an indirect conductimetric assay. J Appl Microbiol 96: 803-809.

SCHITO GC. 2006. The importance of the development of antibiotic resistance in Staphylococcus aureus. Clin Microbiol Infec 1: 3-8

Shahi SK, SHuKla AC, BAJAJ AK, MEDGEly G AND DIKSHIT A. 1999. Broad spectrum antimycotic drug for the control of fungal infection in human beings. Current Science 76: 836-839.

Shopsin B, GOMEz M, MONTGOMERY SO, SMith DH, WAdDingTON M, DODGE DE, Bost DA, RIEHMAN M, NAIDICH S AND KRIESWIRTH BN. 1999. Evaluation of Protein A Gene Polymorphic Region DNA Sequencing for Typing of Staphylococcus aureus Strains. J Clin Microbio 37: 3556-3563.

SiKong L, KONGREONG B, KANTACHOTE D AND SUTTHISRIPOK W. 2010. Photocatalytic activity and antibacterial behavior of $\mathrm{Fe}^{3+}$-doped $\mathrm{TiO}_{2} / \mathrm{SnO}_{2} \mathrm{NPs}$. Energy Res, p. 120-125.

SOLOMON SD, BAHADORY M, JEYARAJASINGAM AV, RUTKOWSKY SA AND BORITZ C. 2007. Synthesis and Study of Silver Nanoparticles. J Chem Educ 84(2): 322325.

SUI ZM, CHEN X, WANG LY, XU LM, ZHUANG WC AND CHAI YC. 2006. Capping effect of CTAB on positively charged Ag nanoparticles. Physica E 33: 308-314.

WRIGHT GD. 2005. Bacterial resistance to antibiotics: Enzymatic degradation and modification. Adv Drug Deliver Rev 57: 1451-1470.

Zhang X, Song H, Yu L, Wang T, Ren X, Kong X, XIE Y AND WANG X. 2006. Surface states and its influence on luminescence in $\mathrm{ZnS}$ nanocrystallites. Lumin 118: 251256. 